\title{
A customized electrical potential difference method for in situ monitoring of propagating cracks using a stochastic algorithm
}

\author{
Evangelos Skrinis ${ }^{1}$, Christos Kalligeros ${ }^{1}$, Antonios Tsolakis $^{2 *}$, and Vasilios Spitas $^{1}$ \\ ${ }^{1}$ Machine Design Laboratory, School of Mechanical Engineering, National Technical University of \\ Athens, Greece \\ ${ }^{2}$ Machine Elements and Vehicle Laboratory, Department of Mechanical Engineering, School of \\ Engineering, University of West Attica, Greece
}

\begin{abstract}
In this paper, a modified direct current potential drop (DCPD) method for real-time measurement of the length, the inclination and the position of cracks is presented. Based on the proposed configuration, it is possible to process the data acquired by continuously measuring the change in the electrical resistance (potential drop) between specific points on the specimen in real time and correlate them with the propagation of the crack and thus identifying its crucial characteristics. Furthermore, many aspects that affect the electromagnetic field inside materials have been identified. In that way the influence of unwanted factors can be significantly reduced which has led to a better understanding of the relation between the implemented voltage values and the fracture itself. Therefore, conclusions are drawn about the structural integrity of any given specimen through a risk assessment after the crack characteristics have been calculated. In order to achieve this, a variety of techniques were implemented including the development of a stochastic algorithm along with a customized experimental layout so as to accomplish high accuracy for the prediction model as well as robustness towards other influencing parameters such as temperature and humidity.
\end{abstract}

Keywords: Crack,Fatigue, Structural health monitoring, Potential drop method, Real-time measurement

\section{Introduction}

Every kind of structure is designed in a way that it fulfills its assigned purpose but furthermore, it allows the prediction of the manner and the time of its failure. For this purpose, a vast range of failure criteria have been developed by engineers for the many different types of applications and circumstances. The existence of cracks is one of the factors that can severely damage any structure's health integrity. That is why the field of fracture mechanics

* Corresponding Author

E-mail address: adtsolakis@uniwa.gr (A. Tsolakis). 
has developed many theories and methods that can be used as a prediction tool for the creation and the propagation of cracks inside any material. Non-destructive techniques constitute a large subcategory of those methods and they can play a crucial role in the safety of any structure and the prolongation of its lifespan. In this paper, the Direct Current Potential Drop (DCPD) method will be analysed as a viable option for the real-time assessment of the structural integrity of electrically conductive laminates. These thin surface structures have a vital role in various fields, such as aerospace and naval engineering and as a result the continuously monitoring of their health against the existence and the propagation of cracks has been the centre of many studies [1-3].

The DCPD technique is a well-known method for measuring the propagation of cracks in challenging environments. According to its basic principle, an electromagnetic field is created inside the selected specimen as a result from the imposition of an already known direct electrical current. The existence of a crack leads to the disturbance of the developed electromagnetic field, in comparison to the uncracked specimen, which is then detected by sensors placed on both sides of the crack and by measuring the potential difference between them. This methodology leads to the designation of the area of the crack and it is described by its high accuracy. However, DCPD is mostly used for generating correlation curves between the applied stress and the crack propagation based on experimental data and measurements, mostly in fatigue experiments on specimens with open cracks and with a priori knowledge of their starting point $[1,2]$. In these experiments the stress intensity factors of a specific component under a specific load are calculated and then used for the prediction of the structure's failure criteria. Notwithstanding the accuracy of the described method, the presumption of the load, the inner microstructure of the specimen as well as the beginning of the crack can lead to either too rigid or too loose failure criteria. So, in this paper, a measuring layout is proposed in which DCPD can be used as a methodology for the live, in-situ monitoring of any kind of crack (Mode I, Mode II and a combination of them) within a designated area and thus providing accurate updates on the health of the structure.

\section{Analysis of the direct problem}

The goal of this paper is to specify and identify the geometrical characteristics of any crack in metal plates utilizing the DCPD method. So, our first milestone was the accurate simulation of the operation of the DCPD technique which would allow the in-depth study of the underlaying phenomena that take place in it. The governing equations of the application of direct current in an electrically conductive plate are the Maxwell equations.

$$
\begin{gathered}
\nabla \cdot \nabla V=0 \\
\mathbf{J}=\sigma \mathbf{E}=-\sigma \nabla V
\end{gathered}
$$

where $\mathrm{V}$ is the potential, $\mathrm{J}$ is the vector of current density, $\sigma$ is the specific material conductivity and $\mathrm{E}$ is the vector intensity of the electrical field. Furthermore, it is assumed that the outer surface of the specimen is not electrically conductive and that the current enters and exits the plate from circles with diameter equal to the diameter of the cables $[1,2]$. Thus, the boundary conditions are formulated as follows:

$$
\begin{gathered}
\mathbf{J} \cdot \mathbf{n}=0 \Rightarrow \frac{\partial V}{\partial \mathbf{n}}=0 \\
\oint_{S_{\text {enter }}} \mathbf{J} \cdot \mathbf{n} d S=-\oint_{S_{\text {exit }}} \mathbf{J} \cdot \mathbf{n} d S=I
\end{gathered}
$$




\subsection{Mathematical analysis - Analytical solution}

Assuming a point source of electrical current and the lower half-space as a propagation medium the following solution arises:

$$
V(r)=\frac{I}{2 \pi r \sigma}
$$

where $\mathrm{V}$ is the potential, $\mathrm{I}$ is the of current, $\sigma$ is the specific material conductivity and $\mathrm{r}$ is the distance from the point of entrance.

However, it is essential to constrain the medium of propagation in order to be able to accurately represent any actual specimen. So, at first, one of the specimen's dimensions was constrained. In the case of the infinite plate with finite thickness, there were noticed some inconsistencies of the previous solution, as far as the boundary conditions were concerned. The solution to this problem was placing virtual mirror current sources in the vertical direction to compensate the effect of the finite thickness in the boundary layers of the specimen [3]. The same technique was followed as the other dimensions are constrained and the resulting potential equation for a metallic plate, with $a / 2$ length, $b / 2$ width and $c / 2$ depth, has the following form [3]:

$$
\begin{aligned}
& V\left(x_{i} y_{i}\right),=\frac{I}{2 \pi \sigma} \sum_{p=1}^{p=2} \sum_{q=1}^{q=2} \sum_{l=-\infty}^{l=\infty} \sum_{m=-\infty}^{m=\infty} \sum_{n=-\infty}^{n=\infty}\left(\frac{1}{\sqrt{r_{A}^{2}+(n c)^{2}}}\right) \\
& r_{A}=\sqrt{\left(x_{i}-(-1)^{p} x_{A}-l a\right)^{2}+\left(y_{i}-(-1)^{q} y_{A}-m b\right)^{2}}
\end{aligned}
$$

This semi-analytical solution of infinite sums converges to a single value after only a few terms. Furthermore, with this methodology it is possible to add features such as electrical anisotropy and heterogeneity to the solution at hand [4].

In addition to this model, a pure mathematical was developed based on the twodimensional solution of the Laplace equation with von Neumann boundary condition with the implementation of complex analysis. More precisely, the Schwarz-Christoffel transformation was used formulate the system of equations that can be used to simulate cracks at many types of thin plates. This solution was developed in MATLAB and the results produced matched the previous solution under the hypothesis that the thickness of the specimen remained small in proportion to its other dimensions. However, due to the complexity of the analytical solution and the computational costs, it was possible to simulate only a limited type of cracks under specific conditions.

\subsection{Computational Solution}

In order to overcome this problem, a computational analysis was performed with the help of the commercial FEA software MAXWELL (Ansys Electronics) which specializes on the solution of the Maxwell equations in many different kinds of materials and boundary conditions. In order to test the validity of the simulation a number of benchmark tests were performed and the results showed a maximum deviation of $3 \%$ both from experimental data and the analytical solutions. This way, by simulating a cracked and an uncracked specimen it was possible to draw conclusions about the DCPD method that were crucial to the development of a successful measuring configuration for the non-destructive testing of a structure's integrity in real time. 


\section{Inverse problem}

The next part in this study focuses on the identification of the geometrical characteristics of the crack in a specimen through the electromagnetic field that is created within it. The crack's features that are required to accurately monitor the structure's health are its:

- Length

- Orientation

- Position

Furthermore, the only information available would be the data collected by the sensors of the measuring layout. The following analysis was based on the potential difference between specific points in the cracked and the uncracked specimen from the many computational experiments that were performed in order to simulate the propagation of a crack.

Before the accurate depiction of the effect of the cracks on the electromagnetic field, it was necessary to identify and analyse any other factors that could affect the values of the electromagnetic field inside any specimen. The isolation of the useful data, from the effect of other attributing factors such as strain, temperature and humidity [5,6], was not a simple task since their contribution must be compensated actively, throughout the operation cycle of any structure. So, the measuring layout had to include sensors such as Wheatstone bridges, at critical points that can provide the necessary information to avoid the misinterpretation of the collected data.

\subsection{Analysis of the crack propagation}

In order to identify the mechanism of the crack propagation an extensive study was initially carried out focusing on the field of Fracture Mechanics and the effects of cracks on the properties of materials. This includes the study of the most predominant theories from the Linear Elastic Fracture Mechanics (LEFM) to the theory of the crack's energy as well as their mathematical hypostasis. Based on them, the failure criteria for the static and dynamic loads were chosen. More specifically the effect of Griffith criterion (Fig. 1) on the yield stress as well as Paris' law (Fig. 1) on the fatigue of materials in corelation with the crack's propagation led to the methodology with which the critical length $\left(\mathrm{a}_{\text {crit }}\right)$ of the defect was identified. This dimension must be detectable by the measuring layout since any crack greater than that could potentially lead to the catastrophe of the mechanism even though its operation load would be within the margins of the traditional failure criteria [6,7]. After the crack has reached this length, the structure should be closely monitored and its condition must be actively revaluated so as to achieve the longest time of safe and non-stopping function as possible.
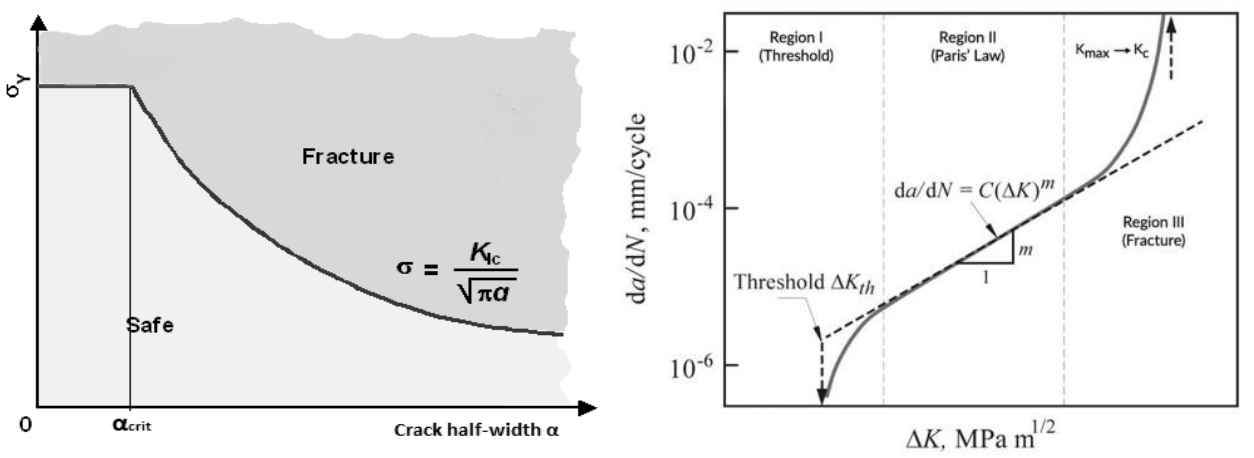

Fig. 1 The Griffith criterion and Paris' law on load and crack's propagation 
It is clear that there is a big variance between characteristics of the measuring layout in terms of sensitivity with regard to each case [6]. To achieve the universal application of the proposed layout through a vast range of different materials, dimensions, components and type of loads, all of its features will be calculated parametrically based on the critical length of the crack. As a result, for every unique application it would be possible to identify the required sensitivity and construct the sensors' configuration from a single experiment on a prototype specimen that would identify the $\mathrm{a}_{\text {crit. }}$.

\subsection{Results of the simulations}

In order to start the computational experiments a square metallic plate with an area of $0.25 \mathrm{~m}^{2}$ from aluminium 7075 with $3 \mathrm{~mm}$ thickness under uniaxial fatigue was selected. This kind of components are used in aerospace applications so the exported data will provide a valid example of a real-world problem rather than a purely theoretical exercise. In this specimen under these specific load conditions the critical length of the crack is $5.6 \mathrm{~mm}[8,9]$.

With this information the experiment was first set up by placing the crack in the middle of the plate perpendicular to the axis X (Fig. 2). Furthermore, the source and the sink of the electrical current were placed on the $\mathrm{X}$ axis with the distance between is arbitrarily chosen to be equal to 20acr. Another important feature that affects the solution of the maxwell equations, is the value of the electrical direct current that is enforced into the specimen and for this DCPD experiment it was 0.5A. As this value it increases, the sensitivity of the measuring layout is improving. However, due to the Joule effect, the electrodes used for the current transmission must also increase their size so that they do not melt [10].
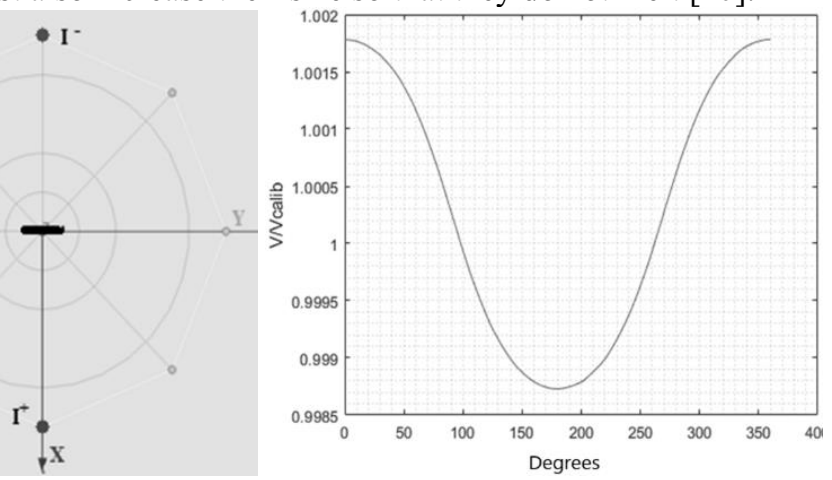

Fig. 2. Configuration of the first computational experiment and the normalized results of the potential

By running the simulation for the cracked and the uncracked specimen and using the results from the last one as a reference, it can be deduced that the effect of the crack in the electromagnetic field is negligible. This can be seen by plotting the electrical potential of the cracked in comparison to the uncracked specimen in the circumference of a circle with diameter equal to four times the critical length of the crack (Fig.2). The maximum deviation of these two values is $0.0018 \%$ which in corelation to the absolute value of the potential difference $(170 \mu \mathrm{V})$, poses a serious issue regarding the sensors' selection. Even after doubling the size of the crack, the overall change in the electromagnetic field in the beforementioned area around the crack is lower than $3 \mu \mathrm{V}$. It is concluded that with this set up, the identification of the critical length of the crack is not possible due to the extreme requirements in terms of technological specifications concerning the sensors' size and sensitivity. 


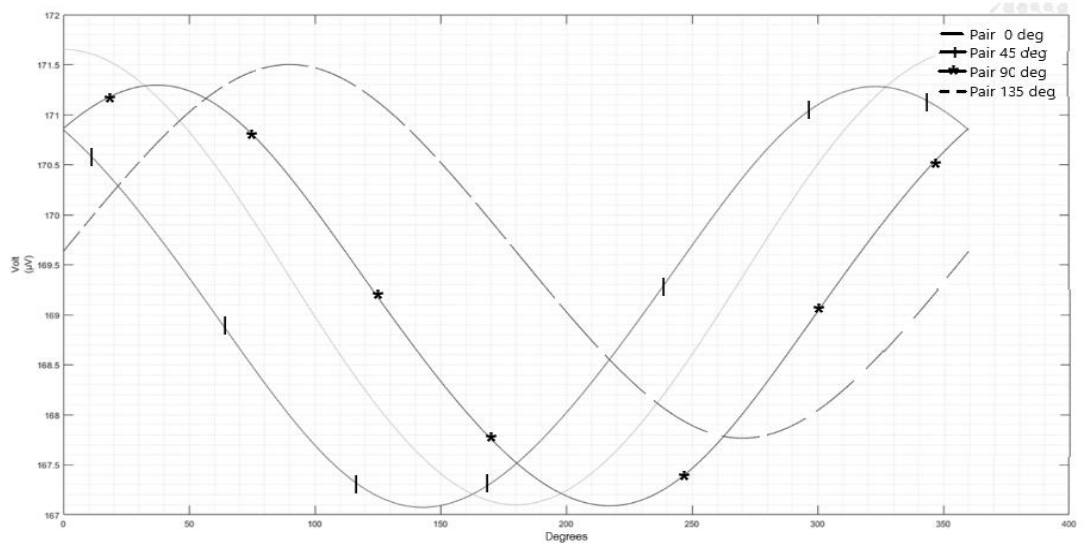

Fig. 3. The potential difference around the area of the crack with different source-sink orientation

Another characteristic of the crack that affects the electromagnetic field in the plate is its inclination. In order to understand this corelation, three new computational experiments are performed with the same set up as far as the crack is concerned. However, the direction of the source-sink pair is changed by a step of 45 degree until a 180-degree turn is completed (Fig. 3). Then the results are plotted again in the circumference of circles, around the crack, with gradually increasing diameters. As it can be seen in the Fig. 3 the potential difference has its greater value when the source-sink pair is perpendicular to the crack in comparison to when it is parallel but only for the small percentage of about $0.02 \%$. As the focus area moves away from the crack these small differences smoothen and the electromagnetic fields end up exactly the same. As a result, it was concluded that by placing pair of electrodes around the crack, is theoretically possible to reach to a conclusion regarding its orientation, but the exceedingly high requirements in terms of the sensitivity and the accuracy of the sensors prevents any real-world application of the chosen layout.

To improve the described situation, it was calculated that it would require an increase of the ampere value by factor of $10^{3}$. So, the consensus was reached that the only thing that would improve the efficiency of the measuring layout would be to place the source-sink pair of the current much closer to the crack. As a result, new computational experiments were designed by positioning the electrode pair perpendicular to the crack at various distances from $20 \%$ to $100 \%$ of the critical length [2]. In order to interpret the results, they were plotted along the imaginary line that connects the source-sink pair (Fig,4). This time the difference between the cracked and the uncracked specimen are much more noticeable. To be more precise the ratio of the two different electrical potentials varies from 1.4 to almost 2. It is obvious that the closer the pair gets to the crack, the differences in the electromagnetic field become conspicuous. However, the decision regarding the position of the current source and sink cannot be made based solely to the sensitivity analysis due to the technological limitations concerning the size of the needed electrodes and sensors.

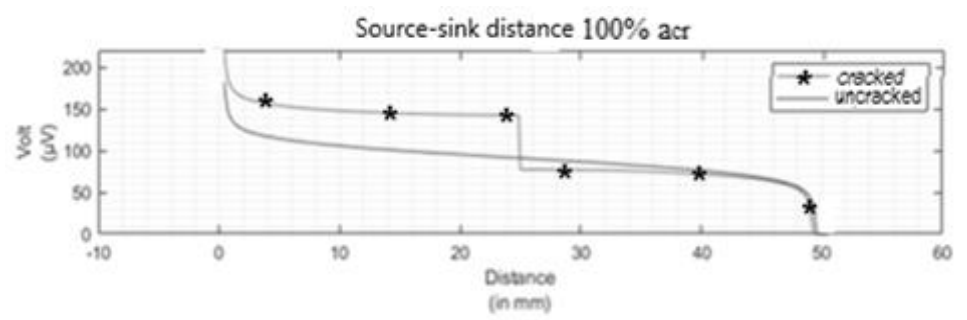

Fig. 4. Electrical potential differences of the cracked and the uncracked specimen 


\section{Results and discussion}

For this reason, the proposed configuration has the form of a grid and the distance between each node is equal to the critical length of the crack. The major advantage of the dense grid that was chosen is that it provides accurate data that can lead to the identification of not only the length but also of the inclination and the position of the crack as well. Each node is used as source or a sink of current or a sensor for the potential difference as the area of interest is swept vertically and horizontally with the use of small and periodical pulses of current. When the electromagnetic field has stabilized, the sensors' measurements are taken. These data, after being compensated for the external factors, they are compared to the values from the uncracked specimen and constitute an indication for the existence of an anomaly in the electromagnetic field. As the process is completed, the results are normalized and the areas that are highlighted are the ones with the higher probability of containing the crack or cracks as it is shown in figure 5.

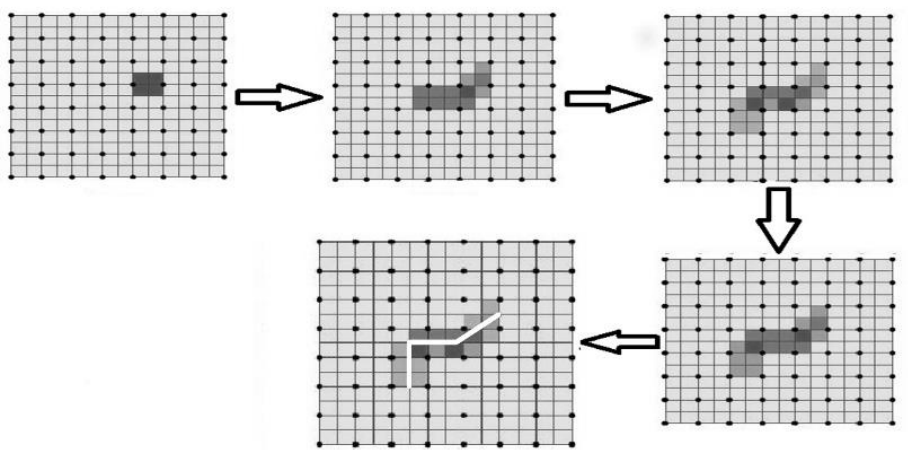

Fig. 5. Probable area of interest of a random crack at a computational experiment after one scan

It is clear that proposed configuration offers advantages that can find application in many fields such as aerospace and naval engineering. The non-invasive configuration of the sensors is a crucial aspect for monitoring the structural integrity of any specimen during its operation cycle. The feature that separates this technique from the majority of the other methods used today is its automated procedure combined with the ability to recognise a crack inside a designated area regardless of its position. As mentioned before, the beginning of the defect is required in most applications of crack monitoring and thus the structure is vulnerable to events that could cause the appearance of the crack somewhere else. Nevertheless, the sensitivity issues, that were faced, enforced a much denser grid of sensors than what it was aimed and thus increasing the complexity and the cost of the whole method. That is why, it is proposed that this configuration should be used not in the whole area of the plate but in the parts that are crucial for the operation of the whole structure. Moreover, its use is suggested in large plates where the increased critical length of the crack makes it much easier for the measuring layout to be implemented.

\section{Conclusion}

In this paper it was shown that the DCPD technique is a valid method for the in-situ monitoring and the safety analysis of electrically conductive materials during a structure's cycle of operation. For this purpose, both an analytical and a computational analysis was performed in the effort to improve the accuracy, sensitivity and robustness of the proposed methodology. A measuring layout was developed concerning thin aluminum plates for the identification of the crack's length, orientation and position. Emphasis should be given to the 
speed and the lack of any a priori knowledge needed for the analysis of a structure's integrity. These two characteristics make the DCPD method a great fit for many scientific experiments and a perfect candidate for real-world applications in the field of non-destructive health monitoring of structures.

\section{References}

1. V. Spitas, C. Spitas, P. Michelis, Real-time measurement of shear fatigue crack propagation at high-temperature using the potential drop technique, Measurement 41, 424-432, 2008.

2. V. Spitas, C. Spitas, P. Michelis, A three-point electrical potential difference method for in situ monitoring of propagating mixed-mode cracks at high temperature, Measurement 43, 950-959,2010.

3. Y.Lu, N.Bowler, J.R.Bowler, Y.Huang, Edge effects in four-point direct current potential drop measurements on metal plates, Journal of physics: Applied Physics, 42, 2009.

4. M.S. Greenhalgh, DC Resistivity modelling and Sensitivity Analysis in anisotropic media, Department of Physics, University of Adelaide, 2008.

5. K.M. Tarnowski, K.M. Nikbin, D.W. Dean, C.M. Davies, Improvements in the measurement of creep crack initiation and growth using potential drop, International Journal of Solids and Structures 134 (2018) 229-248

6. J. Corcora, P. Hooper, C. Davies, P. Nagy, P. Cawley, Creep strain measurement using a potential drop technique, International Journal of Mechanical Sciences 110 (2016) 190-200

7. G.Kaufman, R.L.Moore, P.E.Schilling, Fracture Toughness of structural aluminum alloys, Engineering Fracture Mechanics Vol. 2, Issue 3, pp. 197-210, ,1971.

8. Plane strain fracture toughness (KIC) Data handbook for Metals, U.S. army material and mechanics research center, 1973J.

9. I.Pitropakis, H.Pfeiffer, M.Wevers (2012), Crack detection in aluminium plates for aerospace applications by electromagnetic impedance spectroscopy using flat coil sensors, Sensors and Actuators, A: Physical, 176, 57-63

10. J.Corcoran, C.M.Davies, P.Cawley, A quasi-DC Potential Drop Measurement System for Materials Testing, IEEE Transactions on Instrumentation and Measurement, V. 69, Issue 4, April 2020 